\title{
Der Hypochonder: Das Handbuch für alle, die gerne leiden
}

Ein Hypochonder ist ein Mensch, der sich kratzt, bevor es juckt. Auch wenn Sie dieses Bonmot nicht in dem Buch finden, verspricht dieses eine köstliche, ja höchst vergnügliche Lektüre. Mit Sprachwitz und Wortakrobatik gelingt den Autoren auf heiter ironische Art und Weise eine Aussöhnung mit diesen wahrlich schwierigen Patienten. Die ständige Konfrontation mit solchen gesunden Kranken oder kranken Gesunden ist für Angehörige, aber auch für Ärzte eine schwierige, ja nicht selten unlösbare Herausforderung.

Erschwerend kommt hinzu, dass die moderne Hypochondrie die Nähe zu Modeerkrankungen wie Multi-Chemikalien-Syndrom oder chronischem Müdigkeitssyndrom sucht und gefunden hat. Mit Augenzwinkern werden in dem Buch deshalb auch die krampfhaften, oft pseudowissenschaftlich anmutenden Versuche der medizinischen Wissenschaft beschrieben, solchen Befindlichkeitsstörungen eine Organmaske aufzusetzen. Und mit diesen weltanschaulich geprägten Krankheitsfantasien muss sich dann der Arzt nolens volens auseinandersetzen, was oft nur mit Humor oder auch Ironie gelingen kann.

Auch ist den Autoren erfreulicherweise die schmale Gratwanderung zwischen geziemender Ernsthaftigkeit, wie es das ärztliche Berufsethos auch bei diesem Krankheitsbild erfordert, und einer erfrischend humorvollen Betrachtungsweise gelungen. Das Buch kann daher auch Betroffenen empfohlen werden, solange die Tugend der Selbstironie noch nicht ganz erloschen ist.

Eine unterhaltsame Bereicherung ist das zwischen die Sachinhalte gestreute „Tagebuch eines Hypochonders“. Sicher wird der Leser an vielen Stellen ein Schmunzeln, gelegentlich auch ein herzhaftes Lachen nicht unterdrücken können. Trotzdem gelingt damit auch ein tiefer Einblick in das tragische Erleben eines Betroffenen. Mit Tucholsky empfehle ich Ihnen deshalb: Umarmen Sie dieses Buch, und lesen Sie den „Hypochonder“! Es wird Sie begeistern, denn es verspricht bei diesem traurigen Thema eine wohl dosierte Stimmungsaufhellung, um nicht zu sagen eine psychohygienische Wirkung.

Dr. med. Peter Stiefelhagen -

Erik Heintz, Severin Gröbner, Christian Moser: „Der Hypochonder: Das Handbuch für alle, die gerne leiden“. Südwest Verlag, Mai 2010, ISBN: 3517085308 .

\section{FIBROMYALGIE}

\section{Diagnose ohne Tenderpoint-Testung}

Die Diagnose eines Fibromyalgiesyndroms nach ACR-Kriterien (ACR: American College of Rheumatology) beinhaltet die Druckschmerzhaftigkeit von mindestens 11 von 18 sogenannten Tenderpoints. Da dieses Vorgehen die Fluktuation und den Schweregrad der Schmerzen nicht berücksichtigt und die Tenderpoints zudem oft nicht korrekt untersucht werden, schlägt das ACR jetzt neue Kriterien vor.

Die Patienten sollen gefragt werden, an wie vielen von 19 definierten Regionen sie in der zurückliegenden Woche Schmerzen hatten. Außerdem wird der Symptom Severity Score (SS) ermittelt: Die Ausprägung von kognitiven Problemen, Müdigkeit und nicht erholsamem Schlaf wird jeweils mit 0-3 Punkten bewertet. Bei Vorhandensein weiterer somatischer Beschwerden (Schwindel, Übelkeit, Reizdarm, Depression) können nochmals ma- ximal drei Punkte dazukommen. Ein Fibromyalgiesyndrom liegt vor bei mindestens sieben Schmerzarealen und einem SS von mindestens fünf Punkten bzw. drei bis sechs Schmerzarealen und einem SS von mindestens neun Punkten. Unverändert gilt weiter die Voraussetzung, dass die Beschwerden mindestens drei Monate bestehen müssen und nicht durch eine andere Erkrankung zu erklären sind. BS = Arthritis Care \& Research 2010;62(5):600

\section{ASTHMA BEI KINDERN \\ Pommes und Burger erhöhen das Risiko}

Ob die steigende Prävalenz von Asthma und Allergien mit den Ernährungsgewohnheiten zusammenhängt, wurde in einer internationalen Studie mit 50000 Kindern im Alter zwischen acht und zwölf Jahren untersucht (1995-2005). Das Ergebnis: Kinder, die viel Obst, Gemüse und Fisch essen (mediterrane Diät), erkranken seltener an Asthma. Futtern sie dagegen drei oder mehr Burger pro Woche, ist dies mit einer höheren Asthmaprävalenz assoziiert. Auch Wheezing tritt bei Kindern, die häufig Obst essen, seltener auf. Fisch wirkt sich zumindest in reichen Ländern positiv auf die Wheezing-Prävalenz aus.

KE Thorax 2010;65:516-522 